in which they have been separated from media containing other substances, very likely contain "included" and "occluded" or dissolved impurities.

As has been repeatedly emphasized in papers concerning atomic weight from this laboratory, a solid should be first prepared in such a way as to include or occlude only volatil impurities, and subsequently fused (thus forcing the escape of these volatil impurities), in order to attain purity worthy of serious quantitative consideration in exact work.

\title{
Summary.
}

In this brief paper it has been pointed out that not only inclusion but also solid solution of solvent in crystals is to be feared in precise quantitative work. Such solid solution could neither be easily detected nor easily eliminated. The conclusion is reiterated that the solids obtained from an environment containing other substances, such as a solution, are not safely available for exact quantitative work without further treatment. It is pointed out that these considerations afford an adequate explanation for the anomalous results concerning cadmium sulfate obtained by Perdue and Hulett. Therefore no reason for suspecting an error in the accepted atomic weight of cadmium seems to be indicated by these results.

CAMHRIDGE, MASS.

[Contributions from the Chemical Laboratory of Harvard College.]

THE REFRACTIVE INDEX OF WATER.

By Gregory PaUl Baxter, Laurie Lorne Burgess and Herefrt Wilkens Daudi. Received Apri1 13, 1911.

Investigations in this laboratory upon the refractive indices of salt solutions and the refractive constants of dissolved salts, ${ }^{1}$ have made desirable an exact knowledge of the index of refraction of water at $25^{\circ}$, a temperature for which this constant has never been accurately found, although many determinations have been made at temperatures somewhat above and below this point. In the following investigation determinations were made not only at $25^{\circ}$ but also, for purposes of comparison, at one higher and at one lower temperature.

The accuracy of a determination of the index of refraction of any liquid depends not only upon the sensitiveness and accuracy of the measuring instrument, but also very largely upon the purity of the liquid and the careful regulation of its temperature. It is in the last two respects that many previous investigations have been faulty. In this research these two points received special attention.

The Spectrometer.-The spectrometer was of the Meyerstein type, manufactured by Max Wolz. Its divided circle was graduated in ten-

${ }^{1}$ See following paper. 
minute divisions, and was provided with two verniers on opposit sides of the circle reading directly to ten seconds. It was possible without difficulty, however, to estimate differences of only five seconds. The divided circ'e could be rotated about the prism support, but could be clamped to the prism support in any desired position. Thus errors in the graduation of the scale could be, at least in large part, avoided by averaging results obtained from different portions of the scale. As a matter of fact no differences of this sort of over five seconds were ever discovered. Both telescope and divided circle were provided with micrometer screws for fine adjustment. The prism could be leveled upon the prism table by means of leveling screws. The slit had parallel edges ground perfectly smooth. The telescope was provided with a cross hair which could be illuminated by means of a Gaus octular.

The Prism.-The prism was of solid brass, with a cylindrical opening of $3 \mathrm{~cm}$. diameter, I cm. less than the diameter of the object lenses of telescope and collimator. For the final experiments this prism was heavily silver-plated. Thick plates of glass were cemented upon the prism by means of Canada balsam. These plates of glass had absolutely plane parallel sides as was shown by viewing in the telescope the reflected image of the slit, when illuminated with monochromatic light, and by interposing the prism between telescope and collimator when the latter were in parallel positions. In the first case no multiple images were produced and in the second the image of the slit was not displaced.

The Adjus ment of Temperature.-Since the index of refraction of water for sodium light varies about one unit in the fourth place of decimals per degree, in order to attain an accuracy of one unit in the fifth place of decimals, which was somewhat beyond the accuracy of the spectrometer itself, the temperature must be regulated to one-tenth of a degree Even in a constant temperature room the prism could hardly be expected to remain constant in temperature within so small an amount, owing to heated air currents from the body of the observer and from the lights. Since time is necessary for the accurate setting and reading of the instrument, any method for the simultaneous reading of temperature and setting of the instrument is likely to yield erroneous results, aside from the fact that if the prism contents are changing in temperature, they are likely not to be of uniform density. Hence a device for maintaining the prism and contents at constant temperature was obviously necessary.

For this purpose an air bath was used very similar to those employed by Ruhlmann ${ }^{1}$ and later by Perkin. ${ }^{2}$ This bath was constructed of two perpendicular concentric cylinders of sheet copper, the smaller $14 \mathrm{~cm}$. in diameter, the larger $2 \mathrm{I} \mathrm{cm}$. The space between the cylinders was

1 Pogg. Ann., 132, I86 (1867).

${ }^{2}$ J. Chem. Sac., 6r, 292 (1892). 
divided into three compartments by horizontal copper rings. These compartments were filled with shredded asbestos to ensure better insulation. Openings were cut at suitable places for the introduction of the collimator and telescope, which projected through the walls of the air bath into the interior. The telescope opening was long enough to permit the telescope to swing through an angle of $30^{\circ}$. Both openings were further provided with sliding doors, fitting tightly around the telescope and collimator tubes, by means of which the openings in the air bath could be covered.

Inside the copper jacket were suspended two coils of small lead pipe, containing forty feet in all, through which water at constant temperature could be passed. One of these coils was located above and one below the prism table. The top and bottom of the air bath were made of several thicknesses of asbestos board. The bottom was perforated for the axis of the prism table, the top for two thermometers, one of which was inserted in the prism, but without touching the prism, the other between the prism and the walls of the jacket.

This air bath was supported upon a frame which was absolutely independent of the spectrometer itself, so that straining of the instrument through the very considerable weight of the air jacket could not take place.

Although, when water at constant temperature was circulated through the coils of lead pipe, the temperature of the air bath could be easily adjusted to a tenth of a degree, the two thermometers showed differences in temperature between the prism contents and the air jacket, unless the temperature of the room was very nearly that of the air bath. Under these conditions it seemed probable that the temperature of the prism contents was not uniform. These differences were traced to heat conduction through the metal standard which supported the prism. Hence all the final measurements were made in a room whose temperature was maintained constant always within $2^{\circ}$ and usually within $0.5^{\circ}$ of the temperature of the air bath. Even when the temperatures of the prism contents and the room differed by $2^{\circ}$, those of the prism and air jacket did not differ by more than three-tenths of a degree. Since the temperature of the water was obtained by means of the thermometer immersed in the water in the prism, the accuracy of the adjustment of temperature was evidently sufficient for the purpose.

The Thermometers. - The thermometers were graduated to tenths of a degree and were carefully standardized both by comparison with a thermometer calibrated by the Physikalisch-technisches Reichsanstalt, and by immersion in melting ice and melting sodium sulfate crystals. ${ }^{1}$ Both methods gave essentially identical results.

\footnotetext{
${ }^{1}$ Richards and Wells, Proc. Am. Acad,, 38, 43I (I902).
} 
The Thermostat.--The water which circulated through the coils of the air jacket was brought to constant temperature by passing through a long coil of similar pipe in an electrically heated and regulated water thermostat. In order to provide a stream of water of constant velocity, the water was passed from the main first into a constant-level reservoir. Changes in temperature in the water between the thermostat and the air jacket were avoided by insulating the pipe with a layer of dry cotton.

The Water. -The water was twice distilled, once from alkaline permanganate, and once from very dilute sulfuric acid. In both distillations block-tin condensers were used, and in the final distillation the water was collected in a platinum vessel. Water prepared in this fashion has been frequently tested in this laboratory in atomic weight investigations and has been shown to be free from mineral impurities. In order that it might stand in the prism as short a period as possible, it was introduced into the prism by means of a long-stemmed funnel, after the prism had come to constant temperature in the air jacket. Although, when the water was allowed to stand in the prism, the angle of minimum deviation for sodium light slowly increased, the rate was so slow, about ten seconds in twenty-four hours, that no error from this source was to be feared, especially since less than' one-half hour was normally required for a measurement and since fresh water was used for each new measurement. With a plain brass ptism, and with shellac as the cement this increase in refractivity was nearly three times as rapid, while a nickelplated prism appeared to have no advantage over brass.

That no appreciable quantity of gases was dissolved in this water was shown in one experiment by placing the prism full of water in a vacuum desiccator and exhausting. Only a few minute bubbles appeared, and the indices of refraction were found to be identical before and after this treatment.

The Light.-All adjustments, including the focusing of the collimator and telescope, and all measurements were made with sodium light. Since the difference in the index of refraction of water for the two $D$ lines is not more than two units in the fifth decimal place, which corresponds to a difference in the angle of deviation of only five seconds, obviously the dispersion of the prism needs no consideration. The cross hair of the telescope was always set upon the least deviated side of the line, although the width of the line was too small to measure. The results can be safely assumed to apply to the average wave length of the two sodium lines, 589.3 .

The Measurement of the Angle of the Prism.-The telescope and collimator were first focused for parallel rays, and were adjusted perpendicular to the axis of the instrument in the usual way. After the angle of the prism had had time to come to constancy, it was measured upon the 
spectrometer by the two common methods. By the first method the light was reflected from the collimator into the telescope from the two faces of the prism successively, the collimator and telescope being fixed and the prism rotated. In this way the supplement to the angle of the prism was determined (Method A). By the second method, the prism was fixed in position opposit the collimator, and the telescope was set upon the images of the slit as reflected from the two sides of the prism. In this way an angle double the magnitude of the angle of the prism is found (Method B). Both methods gave essentially the same results, although the accuracy of the second method is twice that of the first. Since it was possible that owing to unequal expansion the prism angle was not constant with changing temperature, measurements of the prism angle were made at various temperatures. No measurable differences could be detected even at the extreme temperatures employed. Furthermore, determinations of the prism angle were made at intervals without disclosing any variation with time.

In making the measurements, from six to ten independent settings of the telescope were made, and the average of these readings was used in the calculations. In only a very few cases was the extreme variation of these readings as much as ten seconds. Fresh observations were then made on different portions of the divided circle.

The Measurement of the Angle of Minimum Deviation.-The prism was first leveled and allowed to come to the temperature of the air jacket. Next it was filled with water as described, and after it had been set at the angle of minimum deviation, the cross hair of the telescope was set upon the sodium line and its position read. After the series of readings of this position had been made, the prism was removed and the direct reading was obtained in the same way. Thus, disturbance of the telescope during the adjustment of the prism was avoided. The operations were then repeated on several different portions of the divided circle.

The Results.-The index of refraction was calculated according to the well known formula,

$$
n=\frac{\sin 1 / 2(D+A)}{\sin 1 / 2 A},
$$

where $D$ represents the angle of minimum deviation and $A$ is the refracting angle of the prism. A positive error of ten seconds in the determination of the refracting angle of the prism produces a difference in the index of refraction of -0.00002 , while a positive error of ten seconds in the determination of the angle of minimum deviation produces a difference of +0.00004 in the refractive index. Assuming a maximum error in each reading of five seconds, the total maximum error in the refractive index is thus 0.00003 . That the error was actually no larger 
than this was evident from the close agreement of the readings in each series, each successive series having been made after the removal of one of the glass plates from the prism and a redetermination of the prism angle.

The angular values recorded in Table I were obtained as follows: 1 From the two vernier readings with the telescope in the position of minimum deviation were subtracted the corresponding two direct readings, and the two differences were averaged. Each ang $e$ in the table is the average of from four to nine such average differences, the extreme variations of which were never over eight seconds and usually less than five seconds. The magnitude of the prism angle was found in a similar way.

TABLE I.

Series I.

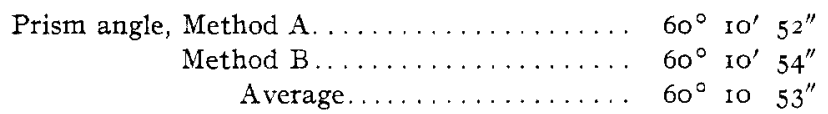

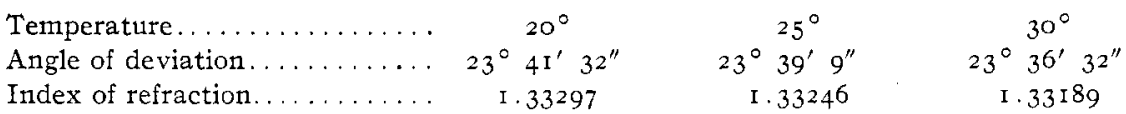

SERIES II.

Prism angle, Method B $60^{\circ}$ I $2^{\prime} 37^{\prime \prime}$

Temperature

$\begin{array}{cc}20^{\circ} & 25^{\circ} \\ 23^{\circ} 42^{\prime} 44^{\prime \prime} & 23^{\circ} 40^{\prime} 17^{\prime \prime} \\ \text { I. } 333302 & \text { I. } 33249\end{array}$

Index of refraction.

I. 33249

SERIES III.

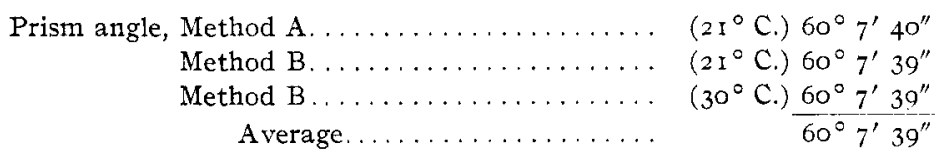

Temperature

$$
\begin{gathered}
23^{\circ} 39^{\prime} 48^{\prime \prime} \\
\text { I. } 33298
\end{gathered}
$$

$$
\begin{gathered}
25^{\circ} 37^{\prime} 28^{\prime \prime} \\
\text { I. } 33248
\end{gathered}
$$

Index of refraction.

SERIES IV.

$$
\begin{aligned}
& \text { Prism angle, Method A........... }\left(30^{\circ} \mathrm{C} .\right) 60^{\circ} 3^{\prime} 28^{\prime \prime} \\
& \text { Method B............. }\left(23^{\circ} \mathrm{C} \text {. } 60^{\circ} 3^{\prime} 3 \mathrm{I}^{\prime \prime}\right. \\
& \text { Method B............. } \quad\left(30^{\circ} \mathrm{C} .\right) \frac{60^{\circ} 3^{\prime} 3 \mathrm{I}^{\prime \prime}}{60^{\circ} 3^{\prime} 30^{\prime \prime}}
\end{aligned}
$$

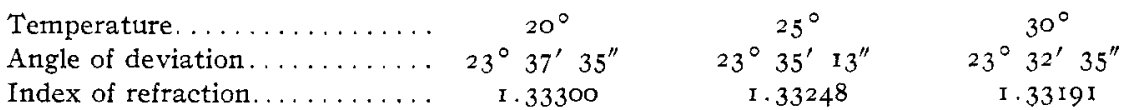

${ }^{1}$ The following experiments were made by L. L. Burgess, 
SERIES V

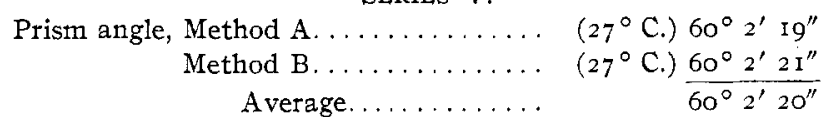

Temperature

Angle of deviation.

Index of refraction.

Temperature.

Average index of refraction.

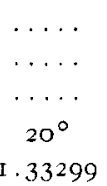

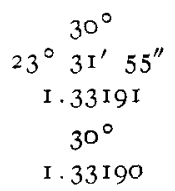

It is interesting to compare these results with those of earlier experimenters. The following data, which are taken from the tables of LandoltBörnstein-Meyerhoffer, are in many cases corrected to the temperature in question, the observations having been obtained at slightly lower or higher temperatures. The averages agree very closely with ours at the same temperatures.

\begin{tabular}{|c|c|c|c|}
\hline & & $20^{\circ}$. & $30^{\circ}$. \\
\hline 1856 & $\ldots \ldots \ldots \ldots$ & I. 33300 & 1.33191 \\
\hline I 867 & van der Willigen............. & I $\cdot 33304$ & .. \\
\hline I 867 & 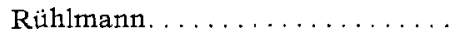 & I.33295 & I. 33200 \\
\hline 1876 & 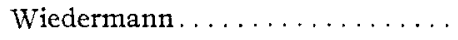 & I. 33291 & $\cdots$ \\
\hline I 880 & 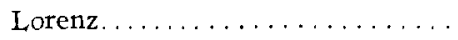 & I. $3330 \mathrm{I}$ & 1.33203 \\
\hline 1885 & $\ldots \ldots \ldots \ldots \ldots \ldots$ & I. 33292 & 1. 33186 \\
\hline 1885 & Kanonnikoff........... & I. 33310 & ... \\
\hline I 888 & Ketteler. ............... & I. 33294 & I $\cdot 33$ I 88 \\
\hline I 889 & Schütt $\ldots \ldots \ldots \ldots \ldots \ldots \ldots \ldots$ & I. 33300 & $\ldots$ \\
\hline 1890 & Bender. . . . . . . . . . . & I. 33287 & . . \\
\hline 1890 & Buchkremer.............. & I.33313 & $\ldots$ \\
\hline $189 \mathrm{I}$ & Brühl. . $\ldots \ldots \ldots \ldots \ldots \ldots \ldots$ & I. 33304 & $\ldots$ \\
\hline I89 I & Röntgen and Zehnder......... & I. 33304 & 1.33194 \\
\hline 1892 & Walter $\ldots \ldots \ldots \ldots \ldots \ldots \ldots$ & 1.33299 & . \\
\hline I 893 & Ruoss..... & I. 33300 & ... \\
\hline 1894 & Simon. & I. 33306 & .. \\
\hline I 894 & 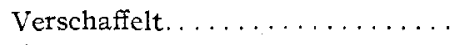 & I.33299 & I. 33196 \\
\hline 1903 & 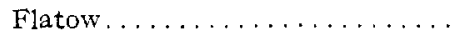 & I. 33300 & $\cdots$ \\
\hline & & & 1.3515 \\
\hline
\end{tabular}

As a check upon the accuracy of these determinations, comparisons of the indices of refraction of water at the three temperatures investigated were made with the Pulfrich refractometer used in the work described in the following paper. The prism and cylinder of this instrument were provided with water jackets through which a stream of water at the desired temperature could be passed. Differences in the angle of emergence could be read by means of a micrometer to o.I'. Differences in index of refraction corresponding to the differences in the readings could be found in tables furnished with the instrument.

The method of experimentation was to allow the temperature of the prism and cylinder to become constant at the desired point. Then the 
cylinder was partly filled with the purest water and after its temperature had become constant several readings of the instrument with sodium light were taken. The water in the cylinder was several times replaced by a fresh portion without ever producing an appreciable difference in reading.

The following differences in the angle of emergence were found in two entirely separate sets of readings, each difference representing from twelve to twenty closely agreeing observations. ${ }^{1}$ The first set of readings was made in an ordinary laboratory in which the temperature fluctuated between 20 and $25^{\circ}$. The second set was made in a room maintained very nearly at the temperature in question.

$$
\begin{aligned}
& \text { TABLE II. }
\end{aligned}
$$

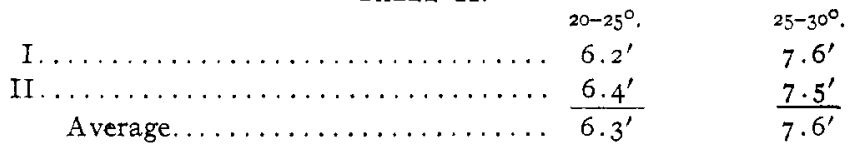

Since with the refractometer employed a difference in the angle of emergence of $1.0^{\prime}$ corresponds to a difference in index of refraction of 0.000077 in the case of a substance having the refractive power of water, the above differences in the angle of emergence correspond to differences in the index of refraction of

$$
\begin{array}{ll}
20-25^{\circ} & 6.3 \times 0.000077=0.000485 \\
25-30^{\circ} & 7.6 \times 0.000077=0.000585
\end{array}
$$

Furthermore, owing to change in the index of refraction of the glass of the prism itself with changing temperature, a positive correction of 0.0000034 per degree is necessary. The corrected values are as follows:

$$
\begin{array}{ll}
20-25^{\circ} & 0.000485+0.000017=0.00050 \\
25-30^{\circ} & 0.000585+0.000017=0.00060
\end{array}
$$

These differences correspond as closely as could be expected with the corresponding differences obtained by the spectrometer method, 0.0005 I and 0.00058 .

In order to correct to vacuum the indices in the table on page 898 , which are of course referred to air at the same temperature, each value must be multiplied by the index of refraction of air at that temperature, referred to vacuum.

$$
\begin{array}{ll}
20^{\circ} & \mathrm{I} .33299 \times \mathrm{I} .000272^{2}=\mathrm{I} .33335 \\
25^{\circ} & \mathrm{I} .33248 \times \mathrm{I} .000267^{2}=\mathrm{I} .33284 \\
30^{\circ} & \mathrm{I} .33190 \times \mathrm{I} .000262^{2}=\mathrm{I} .33225
\end{array}
$$

From the corrected values the specific refraction of water may be calculated by any of the formulas which have been proposed for the purpose. This has been done with the use of the Gladstone and Dale ${ }^{3}$

1 These experiments were made by $\mathrm{H}$. W. Daudt.

${ }^{2}$ Landolt-Börnstein-Meyerhoffer, 689.

s Phil. Trans., r858, p. 887. 
and the Lorentz ${ }^{1}$ and Loren $z^{2}$ formulas. Values for the density of water are taken from the tables of Landolt-Börnstein-Meyerhoffer.

\begin{tabular}{|c|c|c|}
\hline Temperature. & $\frac{n-I}{d}$ & $\frac{n^{3}-1}{n^{2}+2} \frac{I}{d}$. \\
\hline $20^{\circ}$. & $0.33394 \mathrm{I}$ & 0.206256 \\
\hline & 0.333818 & 0.206209 \\
\hline $30^{\circ}$. & 0.333694 & \\
\hline
\end{tabular}

The specific refraction is not constant in either case, diminishing regularly with increasing temperature. ${ }^{3}$ The temperature coefficient is smaller in proportion to the magnitude of the specific refraction in the case of the Lorentz and Lorenz formula. The difference between two successive values of the specific refraction correspond to a difference of 0.00007 in the index of refraction in the case of the Lorentz and Lorenz formula, and of 0.00012 in the case of the Gladstone and Dale formula, while the values for the index of refraction found in this investigation are probably correct within 0.00002 .

As the result of this research the index of refraction of water for sodium light at three temperatures is found to be:

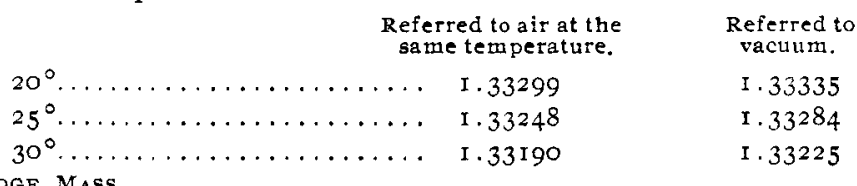

Cambridge, Mass.

[CONtributions from the Chemical Laboratory of Harvard COLlege.]

\section{THE REFRACTIVE POWER OF THE HALOGEN SALTS OF LITHIUM, SODIUM AND POTASSIUM IN AQUEOUS SOLUTION.}

By Gregory P. Baxter, Arthur C. Boylston, Foward Muelier, N. Henry black and PAILIP B. GOODE

Received April 13, IgII.

The refractive power of aqueous solutions of electrolytes has been the subject of numerous researches by many investigators, ${ }^{4}$ and attention has been called to several kinds of regularities. One sort concerns the change in index of refraction of the solution with changing concentration. Another concerns the more or less close application of the Law of Mixtures to the specific refractions of the components of the solution and that of the solution. Still a third regularity has very recently been pointed out by Cheneveau and concerns the change in the index of refraction of the solvent alone as it is diluted with solute.

Wied. Ann., 9, 642 .

${ }^{2}$ Ibid., II, 77 (1880).

${ }^{3}$ Concerning the change in specific refraction with the temperature in the case of organic substances see Falk, This Journal, 3 I, 86 (1909).

* For a very complete bibliography of the subject see Chéneveau, Ann. chim. phys., 8th Series, I2, 384 (1907). 